\title{
Development of transgenic male-sterile rice by using anther-specific promoters identified by comprehensive screening of the gene expression profile database 'RiceXPro'
}

\author{
Maiko Akasaka $^{\dagger 1,2)}$, Yojiro Taniguchi ${ }^{\dagger 3)}$, Masao Oshima ${ }^{3,4)}$, Kiyomi Abe ${ }^{3,5)}$, Yutaka Tabei ${ }^{3)}$ \\ and Junichi Tanaka*1,6) \\ 1) Institute of Crop Science, NARO, 2-1-2 Kannondai, Tsukuba, Ibaraki 305-8518, Japan \\ 2) Present address: Tohoku Agricultural Research Center, NARO, 4 Akahira, Shimo-kuriyagawa, Morioka, Iwate 020-0198, Japan \\ 3) Institute of Agrobiological Sciences, NARO, 2-1-2 Kannondai, Tsukuba, Ibaraki 305-8602, Japan \\ 4) Present address: Tsukuba-Plant Innovation Research Center, University of Tsukuba, 1-1-1 Ten-noudai, Tsukuba, Ibaraki 305-8572, \\ Japan \\ 5) Present address: Biotherapy Institute of Japan Inc., 1-18-2 Sakura, Tsukuba, Ibaraki 305-0003, Japan \\ 6) Graduate School of Life and Environmental Science, University of Tsukuba, 2-1-2 Kannondai, Tsukuba, Ibaraki 305-8518, Japan
}

\begin{abstract}
Because genomic selection is designed for the population breeding of allogamous species, a successive outcrossing system is required for efficient use of genomic selection in autogamous crops, such as Oryza sativa L. (rice). Transgenic and dominant male-sterility is a suitable tool for efficient outcrossing of autogamous crops. Though there have been some reports of dominant male-sterile rice developed using transgenic technology, the flowering habit was substandard. Here, to isolate promoters that, when linked to a lethal gene, induce dominant male-sterility while retaining a good flowering habit, we identified 38 candidate genes with anther-specific expression by using the 'RiceXPro' database. We then evaluated the abilities of the near-upstream regions of these genes to induce male-sterility when linked to the lethal gene barnase and introduced into the rice cultivar 'Nipponbare'. Seven of the 38 promoters induced clear dominant male-sterility; promoters expressed in the later stage of anther development induced male-sterility while retaining better flowering habits when compared to ones expressed in the early stage. These seven promoters could potentially be used to facilitate development of an efficient outcross-based breeding system in rice.
\end{abstract}

Key Words: flowering habits, male-sterility, rice (Oryza sativa L.), outcrossing, barnase, RiceXPro, antherspecific promoter.

\section{Introduction}

Breeding of autogamous crop species commonly starts with bi-parental crossings, and subsequent genetic fixation by selfing, phenotypic screening, and selection of desirable fixed lines. This method has two advantages: 1) the clarity of the relationship between the cross combinations, breeding objectives, and the strategies of screening and selection, and 2) the ease of obtaining high-quality phenotype data for the fixed lines. However, this method has critical disadvantages. Because the breeders have to repeatedly cross already

Communicated by Qian Qian

Received February 21, 2018. Accepted May 24, 2018.

First Published Online in J-STAGE on August 23, 2018.

*Corresponding author (e-mail: tanajun@affrc.go.jp)

${ }^{\dagger}$ These authors contributed equally to this work well-improved materials to breed the best cultivar, the genetic diversity of the breeding population quickly decreases, which in turn leads to less effective breeding. Fujimaki (1980) pointed out the disadvantages of this method as follows: 1) limited use of the full range of available genetic resources, 2) restricted potential for genetic recombination, 3) difficulty in obtaining successive improvements. In fact, the increase in the yields of autogamous crops has slowed drastically since the 1990's (FAOSTAT, http://www.fao.org/ faostat/en/\#data, Tanaka and Tabei 2014).

In contrast, the yield of allogamous maize has grown continuously over the last several decades without signs that it is reaching a peak (USDA National Agricultural Statistics Service, https://www.nass.usda.gov/index.php). The breeding of the parental strains of maize $F_{1}$ cultivars has been driven by recurrent selection-based population improvements, and uses repetitive cycles of selfing and outcrossing 
among genetically diverse populations. This breeding system is powerful because breeders can add selective pressure continuously on the outcrossing populations with many type of genome fragments derived from diverse materials. In addition, genetic recombinations occur very frequently in the population, because most genomic regions are heterozygous. In the maize breeding programs of private companies in mainly US, genomic selection (GS), which uses genomewide markers, has enabled the continuous yield increases. GS-based breeding of livestock animals has also contributed to the dramatic improvement of their traits, especially in the production life of dairy cattle (García-Ruiz et al. 2016, Meuwissen et al. 2001). To use GS effectively for autogamous crop species, however, it is necessary to develop novel breeding systems that can realize effective outcrossbased population breeding.

A previous study has proposed that dominant malesterility with negatively and positively selectable trait markers is an ideal tool for facilitating outcrossing of autogamous crops (Tanaka 2010). Although there are some reports of dominant male-sterility (Ni et al. 2017, Yang et al. 2017), the frequency of emergence of dominant male-sterility is low, and it has been difficult to develop a tightly-linked marker for this trait. In contrast, transgenic technology can provide a very tightly-linked marker if marker genes are introduced with the dominant male-sterility gene into the genome by the same vector construct. Since there is no counterpart sequence of the introduced sequence on the homologous chromosome, there is very little risk of linkage break-up.

The development of dominant male-sterility is not technologically difficult when we employ a construct containing an anther-specific promoter driving a lethal gene such as barnase, encoding ribonuclease from Bacillus amyloliquefaciens (Acc. No. M14442, EC 3.1.27, Paddon and Hartley 1985). This type of dominant male-sterility has been developed in many plants, such as oilseed rape (Brassica napus L., Mariani et al. 1990), wheat (Triticum aestivum, De Block et al. 1997), oilseed mustard (Brassica juncea, Jagannath et al. 2001), maize (Zea mays, Sun et al. 2008), eggplant (Solanum melongena, Cao et al. 2010), pine (Pinus radiata, Zhang et al. 2012) and eucalyptus (Eucalyptus occidentalis, Zhang et al. 2012), and pelargonium (Pelargonium zonale, García-Sogo et al. 2012). There are some reports on development in rice, however, in many cases, the developed recombinants have problems in flowering habits, such as flowering rate and flowering time (Abe et al. 2018, Lu et al. 2000). Because this tendency is also found in non-transgenic male-sterile rice derived by mutation (Tamaru 1994), it is presumed that this tendency is a general issue of male-sterility in rice. Since pollen fertilization ability of rice is lost within $30 \mathrm{~min}$ (Song et al. 2001), excellent flowering characteristics is a key for efficient outcrossing fertility in rice. To obtain practical male-sterility by transgenic technology, the timing and organ-specificity of lethal gene expression are important. Therefore, the development of a highly anther-specific promoter is desired.
The rice genome was sequenced completely with extremely high precision prior to the genomes of other crops (International Rice Genome Sequencing Project 2005), and databases for genomic sequences and genes (Sakai et al. 2013), expression profiles of genes (Kawahara et al. 2016, Sato et al. 2011), and detected QTLs (Yonemaru et al. 2010) have been published. Here, comprehensive screening of the expression profile database 'RiceXPro' was conducted to identify the best anther-specific promoters. Thirty-eight genes specifically expressed in anthers were identified, and the ability of their near-upstream sequences to induce dominant male-sterility with desirable flowering habit was evaluated.

\section{Materials and Methods}

The rice cultivar 'Nipponbare' was used as wild type in all experiments in this study.

\section{Comprehensive screening for anther-specific promoters in 'RiceXPro'}

Fig. 1 shows the flow of screening for Anther- $\underline{\text { Specific }}$ Promoters (ASPs) in this study. First, we accessed the data set designated as RXP_000 in the rice expression profile database RiceXPro (Sato et al. 2011, 2013, http://ricexpro. dna.affrc.go.jp) published by the National Institute of Agrobiological Sciences and screened it five times (100-300 genes per screening) according to the intensity of expression at four stages of anther development (phases 1-4 in RiceXPro). Genes with expression profiles in the following five categories were identified: I) very high anther-to-pistil expression ratio; II) extremely high expression in phase 4; III) high expression in phase 2 or 3 ; IV) moderate expression peaking in phase 3 or 4 , and $V$ ) high expression peaking in phase 3 or 4 . To identify genes with anther-specific expression, we then screened the combined list of the above genes (overlaps removed) for no or extremely low expression in other tissues (leaf blade, leaf sheath, root, stem, panicle, lemma/palea, ovary, embryo, and endosperm) based on visual appearance in the 'Raw Signal Intensity Bar Graph'. Thus, a subset of candidate genes with anther-specific expression were identified.

We analyzed the sequences of these anther-specific genes by using the annotation databases Rice TOGO Browser (Nagamura et al. 2011, http://agri-trait.dna.affrc.go.jp) and RAP-DB (Sakai et al. 2013, http://rapdb.dna.affrc.go.jp) to select those where 1) the distance to the gene upstream was $>800 \mathrm{bp}$, and 2) the near-upstream sequences (containing promoter region) did not have many restriction enzyme sites or GC-rich repeat regions. Finally, we selected 38 nearupstream sequences of the genes that fulfilled the abovementioned conditions, and labelled the sequences as ASPs in all five categories, respectively (Table 1).

\section{Amplification and modification of ASP sequences}

ASP fragments were obtained by PCR amplification 


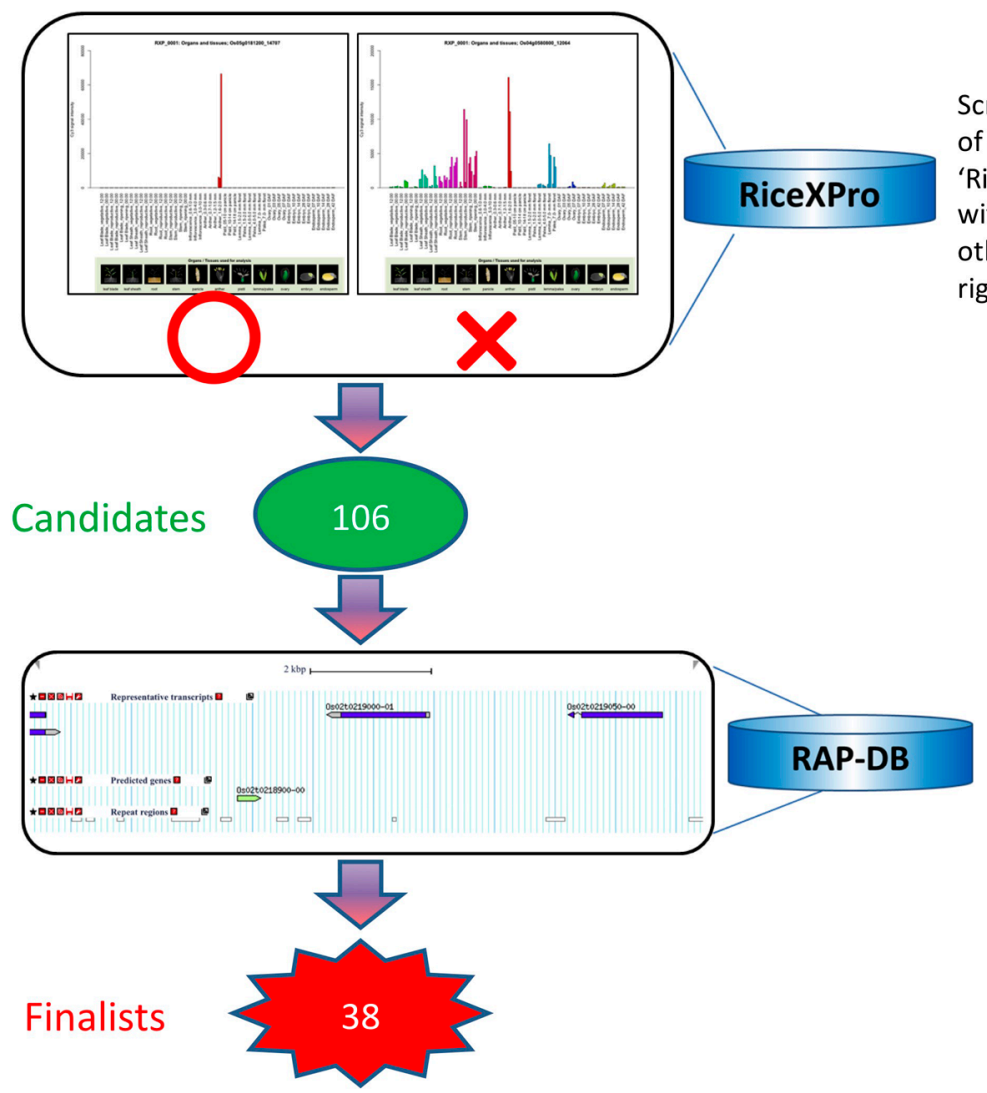

Screen list of genes showing very high levels of expression in anthers relative to pistils in 'RiceXPro' (Sato et al. 2011), for candidates with no or extremely low expression levels in other tissues (e.g., gene in left panel, not right panel).

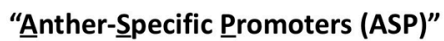

Check the genome information with 'Rice TOGO Browser' (Nagamura et al. 2011) and 'RAP-DB' (Sakai et al. 2013) to screen for candidates with

1) a large gap from upstream genes.

2) not many restriction enzyme sites or $\mathrm{GC}$-rich repeat regions in the upstream sequences.

Fig. 1. Overview of screening for anther-specific promoters. Screen list of genes showing very high levels of expression in anthers relative to pistils in 'RiceXPro' (Sato et al. 2011) for candidates with no or extremely low expression levels in other tissues (e.g., gene in left panel, not right panel). Check the genome information with 'Rice TOGO Browser' (Nagamura et al. 2011) and 'RAP-DB' (Sakai et al. 2013) to screen for candidates with 1) a large gap from upstream genes. 2) not many restriction enzyme sites or GC-rich repeat regions in the upstream sequences.

from rice genomic DNA extracted from seedlings by using diatomaceous earth and a spin filter (Tanaka and Ikeda 2002) or a DNeasy Plant Mini Kit (Qiagen, Venlo, Netherlands). Primer sets for PCR amplification were designed based on the ASP candidate sequences with additional $X b a \mathrm{I}$ and BamHI sites (Supplemental Table 1). PCR amplifications were performed using a PrimeSTAR (TaKaRa, Shiga, Japan) or KOD FX Neo (Toyobo Life Science, Osaka, Japan) with $0.35 \mathrm{ng} / \mu \mathrm{L}$ final concentration of template DNA, $0.4 \mathrm{mM}$ dNTPs, and $0.3 \mu \mathrm{M}$ each primer. Touchdown PCR (Don et al. 1991) with PrimeSTAR DNA polymerase was performed as follows: $5 \mathrm{~min}$ at $94^{\circ} \mathrm{C} ; 34$ cycles of $30 \mathrm{~s}$ at $94^{\circ} \mathrm{C}, 60 \mathrm{~s}$ at annealing temperature (described below), and $30 \mathrm{~s}$ at $72^{\circ} \mathrm{C} ; 10 \mathrm{~min}$ at $72^{\circ} \mathrm{C}$. The annealing temperature was $62^{\circ} \mathrm{C}$ in the first cycle; lowered by $0.5^{\circ} \mathrm{C}$ per cycle during cycles 2 to 14 ; and retained at $55^{\circ} \mathrm{C}$ for the last 20 cycles. PCR with KOD FX Neo DNA polymerase was performed as follows: $2 \mathrm{~min}$ at $94^{\circ} \mathrm{C}, 32$ cycles of $10 \mathrm{~s}$ at $98^{\circ} \mathrm{C}$, and $5 \mathrm{~min}$ at $68^{\circ} \mathrm{C}$. ASP304 sequence was obtained by nested PCR; the PCR product from the first primer set was used as a template. Sequences of ASP102 and ASP114 were synthesized by a gene synthesis service (GenScript Inc., Piscataway, NJ, USA) (Supplemental Fig. 1). PCR products of ASPs were purified with a QIAquick Gel Extraction Kit (Qiagen), and adenine base was added to the $3^{\prime}$ end by using EX Taq polymerase (TaKaRa). PCR products were subcloned into pGEM-T Easy vector (Promega Corporation, Madison, WI, USA) and their sequences were confirmed by Sanger sequencing. All XbaI, BamHI, AscI, MluI, and EcoRI restriction enzyme sites in the ASP sequences were mutagenized by PCR using the PrimeSTAR Mutagenesis Basal Kit (TaKaRa) or designed primers (Table 2, Supplemental Table 1).

\section{Vector construction and rice transformation}

The binary vector used in this study was constructed using a pZH2Bi-KXB vector (Kuroda et al. 2010, Fig. 2). Each ASP sequence was connected with the extracellular ribonuclease gene, barnase to drive anther-specific cell death. To cancel out the influence of leaky expression of the barnase gene in non-anther tissues, we inserted a barstar cassette in the same construct; this cassette harbored the Cauliflower mosaic virus (CaMV) $35 \mathrm{~S}$ promoter, a barnasespecific inhibitor gene "barstar" (Abe et al. 2018), and a double terminator (DT) consisting of the CaMV $35 \mathrm{~S}$ terminator and nos terminator (Luo and Chen 2007). Each 


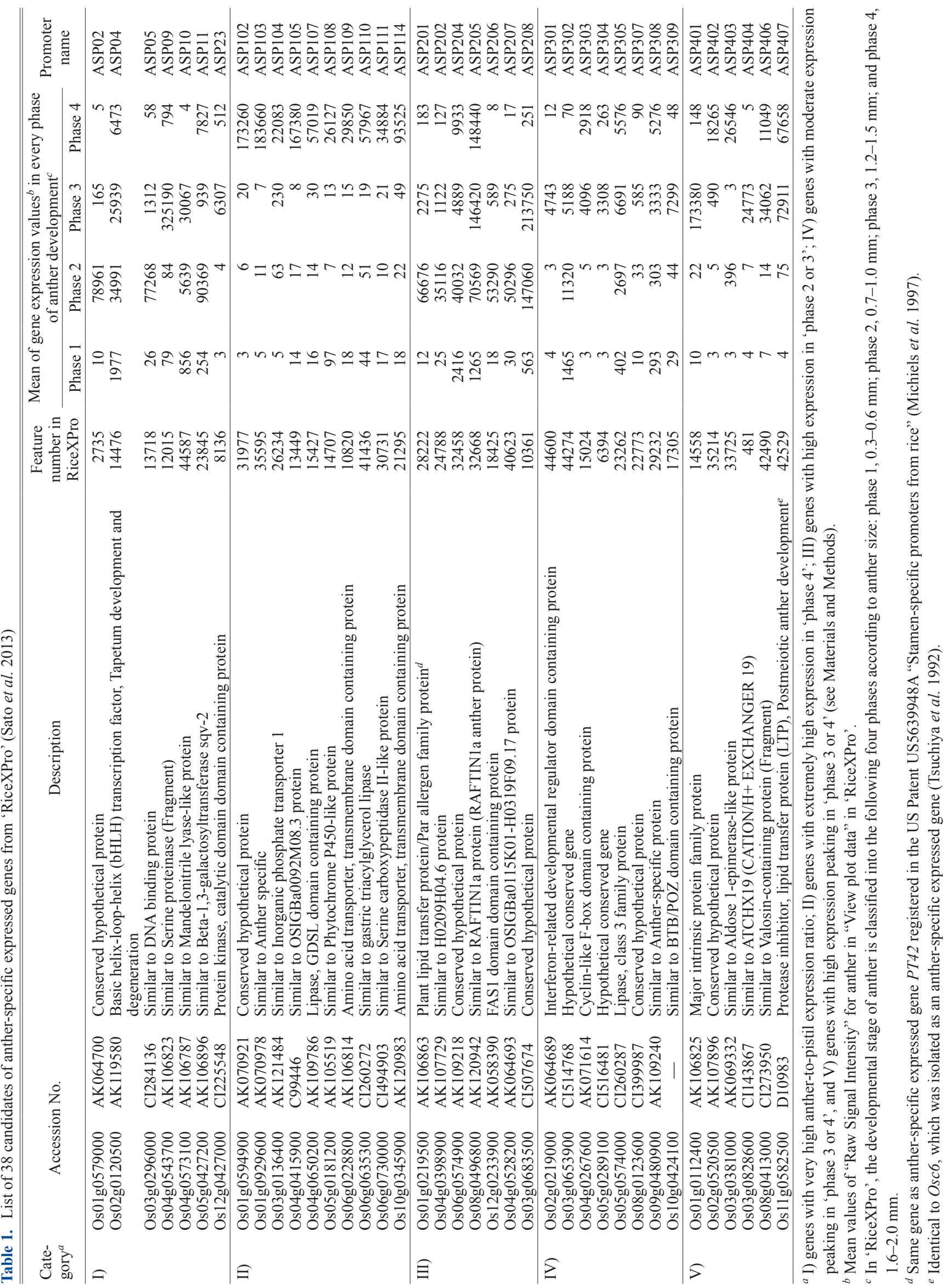


Table 2. Information on the promoter regions of the anther-specific expressed genes

\begin{tabular}{|c|c|c|c|c|c|}
\hline \multirow{2}{*}{$\begin{array}{l}\text { Promoter } \\
\text { name }\end{array}$} & \multirow{2}{*}{$\begin{array}{c}\text { Amplified } \\
\text { sequence size } \\
\text { (bp) }\end{array}$} & \multirow[b]{2}{*}{ Region of amplified promoter ${ }^{a}$} & \multicolumn{3}{|c|}{ Mutagenesis } \\
\hline & & & $\begin{array}{l}\text { Mutagenized } \\
\text { restriction enzyme site }\end{array}$ & $\begin{array}{c}\text { Position of } \\
\text { mutagenesis }^{b}\end{array}$ & Modifications \\
\hline ASP02 & 1202 & chr01:22,398,039..22,400,110 (- strand) & & & \\
\hline ASP04 & 2004 & chr02:1,076,181..1,078,184 (- strand) & & & \\
\hline ASP05 & 1534 & chr03:10,365,265..10,366,804 (- strand) & & & \\
\hline ASP09 & 926 & chr04:27,221,751..27,222,679 (+ strand) & & & \\
\hline ASP10 & 502 & chr04:28,869,880..28,870,381 (+ strand) & MluI & 2 & $\mathrm{~A} \rightarrow \mathrm{T}$ \\
\hline ASP11 & 1070 & chr05:20,947,056..20,948,125 (- strand) & & & \\
\hline ASP23 & 1889 & chr12:13,601,137..13,603,025 (- strand) & $X b a \mathrm{I}$ & 1502 & $\mathrm{~A} \rightarrow \mathrm{C}$ \\
\hline ASP102 & 1963 & chr01:23,312,537..23,314,499 (- strand) & & & \\
\hline ASP103 & 907 & chr01:40,798,447..40,799,353 (- strand) & & & \\
\hline ASP104 & 835 & $\operatorname{chr} 03: 2,013,430 . .2,014,264$ (- strand) & BamHI & 565 & $\mathrm{G} \rightarrow \mathrm{A}$ \\
\hline ASP105 & 1325 & chr01:40,798,447..40,799,353 (- strand) & & & \\
\hline ASP107 & 1626 & chr04:33,134,195..33,135,820 (- strand) & & & \\
\hline ASP108 & 921 & chr05:4,884,501..4,885,421 (+ strand) & & & \\
\hline ASP109 & 1242 & chr06:6,695,017..6,696,258 (- strand) & BamHI & 1192 & $\mathrm{~A} \rightarrow \mathrm{T}$ \\
\hline ASP110 & 1613 & chr06:25,755,592..25,757,204 (- strand) & & & \\
\hline ASP111 & 951 & chr06:31,109,826..31,110,776 (+ strand $)$ & & & \\
\hline ASP114 & 1443 & chr10:10,356,456..10,357,898 (+ strand) & BamHI & 203/1018 & $\mathrm{TC} \rightarrow \mathrm{AT} / \mathrm{C} \rightarrow \mathrm{G}$ \\
\hline ASP201 & 1951 & chr01:6,539,046..6,540,996 (+ strand $)$ & BamHI & 1036 & $\mathrm{~T} \rightarrow \mathrm{G}$ \\
\hline ASP202 & 2191 & chr04:19,711,441..19,713,631 (- strand) & & & \\
\hline ASP204 & 2276 & chr06:22,328,886..22,331,163 (- strand) & & & \\
\hline ASP205 & 2214 & chr08:24,531,119..24,533,332 (+ strand $)$ & & & \\
\hline ASP206 & 2272 & chr12:7,328,101..7,330,372 (+ strand) & & & \\
\hline ASP207 & 1349 & chr04:26,390,979..26,392,327 (+ strand) & & & \\
\hline ASP208 & 1991 & chr03:27,230,812..27,232,802 (+ strand) & & & \\
\hline ASP301 & 2072 & chr02:6647952..6645881 (- strand) & BamHI & 2008 & $\mathrm{~A} \rightarrow \mathrm{T}$ \\
\hline ASP302 & 2411 & chr03:25470682..25468272 (- strand) & $X b a \mathrm{I}$ & 1257 & $\mathrm{TTCT} \rightarrow \mathrm{CCGC}$ \\
\hline ASP303 & 2415 & chr04:11064467..11062053 (- strand) & BamHI & 780 & $\mathrm{CC} \rightarrow \mathrm{AA}$ \\
\hline ASP304 & 2158 & chr05:12570317..12572474 (+ strand) & & & \\
\hline ASP305 & 2483 & chr05:28595736..28598218 (+ strand) & & & \\
\hline ASP307 & 2416 & chr08:1293225..1290810 (- strand) & & & \\
\hline ASP308 & 2431 & chr09:18454621..18457051 (+ strand) & & & \\
\hline ASP309 & 2487 & chr10:15033604..15036090 (+ strand) & $X b a \mathrm{I}$ & 646 & $\mathrm{TAG} \rightarrow \mathrm{CGC}$ \\
\hline ASP401 & 1394 & chr01:648121..646728 (- strand) & & & \\
\hline ASP402 & 1919 & chr02:18954598..18956516 (+ strand) & & & \\
\hline ASP403 & 1851 & chr03:15104287..15102437 (- strand) & & & \\
\hline ASP404 & 2053 & chr03:34800840..34802892 (+ strand) & & 781 & $\mathrm{TA} \rightarrow \mathrm{AC}$ \\
\hline ASP406 & 1926 & chr08:19762731..19760806 (- strand) & & & \\
\hline ASP407 & 1565 & 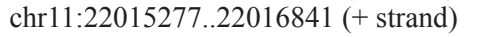 & $X b a \mathrm{I}$ & 896 & $\mathrm{~T} \rightarrow \mathrm{A}$ \\
\hline
\end{tabular}

a Sequence position on the 'Nipponbare' IRGSP-1.0 reference genome.

${ }^{b}$ Counted from the beginning of the promoter

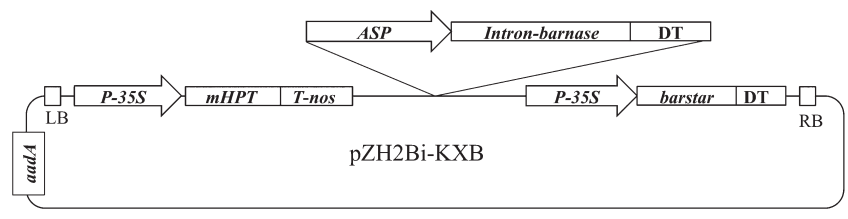

Fig. 2. Binary vector used in this study, pZH2Bi-KXB. ASP, antherspecific expressed gene promoter region; aadA, spectinomycin resistance protein; $P-35 S$, CaMV $35 S$ promoter; $m H P T$, modified hygromycin phosphotransferase; T-nos, nopaline synthase terminator; DT, $35 S$ and nos double terminator; LB, T-DNA left border; RB, T-DNA right border.

ASP sequence in pGEM vector was digested by $X b a \mathrm{I}$ and BamHI, and inserted upstream of barnase in the pZH2Bi$\mathrm{KXB}$ vector. As a control, a construct using the BoA9 promoter, which has already been confirmed to induce male- sterility when connected upstream of barnase (Abe et al. 2018, Konagaya et al. 2008), was constructed in the same manner as for the ASP constructs.

The binary vectors were introduced into Agrobacterium tumefaciens strain EHA105, and then used for transformation under the culture conditions described previously (Ozawa and Takaiwa 2010). About 20 individuals per construct were produced and cultivated in the simplified Biotron Breeding System (sBBS) (Tanaka et al. 2016) under condition of $27^{\circ} \mathrm{C}$ during the 10 -h-light period $(230 \mu \mathrm{mol}$ photons $\mathrm{m}^{-2} \mathrm{~s}^{-1}$, from 7:00 to $\left.17: 00\right), 25^{\circ} \mathrm{C}$ during the $14-\mathrm{h}$ dark period, and $600 \mathrm{ppm} \mathrm{CO}_{2}$.

\section{Observation of anther shapes and pollen}

Spikelets were sampled from the panicles a few days after heading. Three or more individuals per construct were investigated. Anthers were stained overnight at room 
temperature according to Alexander (1969). The presence or not of active pollen and the degree of pollen staining were examined using a Microphot-FXA EPI-FL3 microscope (Nikon, Tokyo, Japan).

\section{Checking the sterility characteristics of transformants}

For each individual transformant, seed settings in a main culm panicle were counted to confirm the sterility. We judged "sterility" as fewer than three set seeds, because cross pollination can happen in a close planting under sBBS conditions. The subset of transformants with confirmed sterility and stable growth were pruned back and re-grown in a closed greenhouse or under sBBS again for confirmation of male-sterility by female-fertility test. The upper parts of spikelets in some panicles of transformants were cut off, and put in a bag together with the flowering panicles of the pollen parent (i.e., wild type). The bags were shaken every 30 min under sBBS, or every $1 \mathrm{~h}$ in a closed greenhouse, between 11:30 and 14:30 over the period of flowering. For each transformant, after about one month, the panicles were harvested and the number of seeds was counted; then, for each construct, the percentage of sterility was calculated as (number of investigated plants - number of fertile plants/ number of investigated plants) $\times 100$.

\section{Investigation of flowering habits}

Three of the constructs (ASP108, ASP208, and ASP304, Table 1) which produced transformants with male-sterility, female fertility, and normal growth, were used to produce transformants again, and compared to equivalent constructs containing the $B o A 9$ promoter instead of the ASP. About 10 individuals per construct were cultivated under sBBS, and their main culm panicles were investigated from their heading date onwards: opened spikelets were counted every hour from 9:00 to 17:00 until an opened spikelet was not observed for over three days. The following phenotypes were compared between transformants: 1) number of days between heading and flowering; 2) number of days between the onset of flowering and the flowering peak; 3 ) flowering period; 4) flowering rate from 13:00 to 15:00 (the peak flowering time in wild type); and 5) flowering rate (number of opened spikelets/all spikelets) (Supplemental Fig. 2).

\section{Results}

\section{In silico screening of ASPs from RiceXPro database}

In our comprehensive series of screens of the rice expression profile database RiceXPro, we identified a total of 106 genes based on (a) very high expression in anthers relative to pistils and (b) no or extremely low expression in other tissues. The number of genes in the five categories of expression during anther development (see Materials and Methods for details) were as follows: category (I), 23 genes; category (II), 14 genes; category (III), 8 genes; category (IV), 44 genes; category (V), 22 genes; 5 overlaps were removed. We then performed a further screen using TOGO
Browser and RAP-DB to identify which of these antherspecific genes had the most potentially useful upstream sequences for use in expression cassettes. As a result, we identified a total of 38 ASPs to use in further experiments: 7 from category (I), 10 from category (II), 7 from category (III), 8 from category (IV), and 6 from category (V) (Table 1). The flow chart of screening used in this study is shown in Fig. 1.

\section{Production of transformants and phenotype screening}

Each ASP was cloned and some were mutagenized by PCR to remove restriction enzyme sites as necessary (Table 2, Supplemental Fig. 1). The BoA9 promoter, which is known to induce male-sterility when directing expression of barnase gene (Abe et al. 2018, Konagaya et al. 2008), was cloned and used as a control. Each ASP or BoA9 promoter was connected with barnase, to construct binary vectors composed of the following three cassettes aligned in tandem: the hygromycin resistance cassette, anther-specific barnase gene-expressing cassette, and CaMV $35 S$ promoterdriven barstar gene-expressing cassette (Fig. 2). Using the prepared construct, rice was transformed via the Agrobacterium method.

Constructs containing ASP103 or ASP307 failed to regenerate plants from hygromycin-resistant calli after selection. Regenerated plants were obtained from six ASP constructs (ASP05, ASP09, ASP10, ASP107, ASP114, and ASP303), but they did not grow normally and most of them died immediately after transplantation. For the remaining $30 \mathrm{ASP}$ constructs, transformants grew normally until heading. However, for 18 of these constructs, most individuals suddenly died around the time of heading, so $\leq 10$ out of $\sim 20$ regenerated individuals could be investigated for sterility (Table 3 ). Finally, for a total of 12 ASPs, namely ASP04, ASP108, ASP110, ASP111, ASP204, ASP207, ASP208, ASP304, ASP305, ASP308, ASP401, and ASP407, we confirmed that most of the regenerated individuals grew normally.

\section{Phenotypic features of transformants \\ 1) Anther and pollen}

The anthers of transformants harboring ASP04, ASP204, ASP206, ASP207, ASP208, ASP302, or ASP407 were white and degenerated, and pollen grains could hardly be observed inside (Fig. 3). Conversely, the anthers of transformants produced by the other 23 constructs were yellow, and pollen grains were observed inside them, as in wild type. 2) Sterility characteristics

Transformants harboring ASP04, ASP204, ASP206, ASP207, ASP208, ASP302, or ASP407 were observed to have no pollen grains (i.e., complete sterility) in all individuals (Table 3). In addition, transformants harboring ASP108, ASP109, ASP301, or ASP304 were sterile in most individuals even though pollen grains were observed in their anthers (Table 3); these pollen grains stained with Alexander's solution, but were inferior to wild type in terms of their amount and fullness (Fig. 3). We judged the 
Table 3. Phenotypic features and sterility characteristics of transformants

\begin{tabular}{|c|c|c|c|c|c|}
\hline $\begin{array}{l}\text { Phase with maximum } \\
\text { expression in anther }^{a}\end{array}$ & Construct & Pollens & $\begin{array}{l}\text { No. of investigated } \\
\text { plants }^{b}\end{array}$ & No. of fertile plants & Sterility (\%) \\
\hline \multirow[t]{9}{*}{ Phase 2} & ASP02 & unidentified & 3 & 3 & 0 \\
\hline & $\underline{\mathrm{ASP} 04}$ & unidentified & 14 & 0 & 100 \\
\hline & $\overline{\overline{\text { ASP11 }}}$ & unidentified & 2 & 1 & 50 \\
\hline & ASP201 & identified & 10 & 3 & 70 \\
\hline & ASP202 & identified & 3 & 2 & 33 \\
\hline & ASP204 & unidentified & 20 & 0 & 100 \\
\hline & $\overrightarrow{\text { ASP206 }}$ & unidentified & 4 & 1 & 75 \\
\hline & ASP207 & unidentified & 17 & 0 & 100 \\
\hline & $\overline{\mathrm{ASP} 302}$ & unidentified & 1 & 0 & 100 \\
\hline \multirow[t]{9}{*}{ Phase 3} & ASP23 & identified & 5 & 5 & 0 \\
\hline & ASP208 & unidentified & 17 & 0 & 100 \\
\hline & $\overline{\overline{\text { ASP301 }}}$ & identified & 5 & 1 & 80 \\
\hline & ASP304 & identified & 18 & 1 & 94 \\
\hline & $\overline{\mathrm{ASP} 305}$ & identified & 21 & 19 & 10 \\
\hline & ASP309 & identified & 3 & 1 & 67 \\
\hline & ASP401 & identified & 16 & 7 & 56 \\
\hline & ASP404 & identified & 1 & 0 & 100 \\
\hline & ASP406 & identified & 2 & 2 & 0 \\
\hline \multirow[t]{14}{*}{ Phase 4} & ASP102 & identified & 5 & 3 & 40 \\
\hline & ASP104 & identified & 4 & 4 & 0 \\
\hline & ASP105 & identified & 3 & 3 & 0 \\
\hline & ASP108 & identified & 18 & 1 & 94 \\
\hline & ASP109 & identified & 9 & 1 & 89 \\
\hline & ASP110 & identified & 16 & 10 & 38 \\
\hline & ASP111 & identified & 14 & 5 & 64 \\
\hline & ASP205 & identified & 1 & 1 & 0 \\
\hline & ASP308 & identified & 19 & 18 & 5 \\
\hline & ASP402 & identified & 3 & 0 & 100 \\
\hline & ASP403 & identified & 1 & 1 & 0 \\
\hline & ASP407 & unidentified & 19 & 0 & 100 \\
\hline & BoA9 & unidentified & 20 & 0 & 100 \\
\hline & Nipponbare & identified & 2 & 2 & 0 \\
\hline
\end{tabular}

Single underlines indicate the representative constructs that typically generate pollen-producing sterile transformants.

Double underlines indicate the representative constructs that typically generate pollen-less sterile transformants (Fig. 3).

${ }^{a}$ Categorization according to "Mean gene expression values" in Table 1.

${ }^{b}$ Number of individuals with normal growth from about 20 regenerated individuals.

transformants derived from the 19 other ASP constructs to be non-sterile or unclassifiable because numerous set seeds were observed or too few individuals $(\leq 3)$ survived past heading, respectively. Finally, we identified seven ASPs, namely ASP04, ASP108, ASP204, ASP207, ASP208, ASP304, and ASP407, as promising promoters inducing normal growth and effective sterility phenotypes.

\section{3) Confirmation of male-sterility by female-fertility test}

Artificial crossing with wild-type pollen demonstrated that all individuals derived from the above seven ASP constructs showed female-fertility (i.e., cross-fertility; Table 4); with the exception of one individual derived from the ASP108 construct. Because transformants derived from these seven constructs produced almost no seeds by selfing (Table 3), but showed female fertility, we judged them to show male-sterility, presumably induced by the respective ASP and barnase.

\section{Flowering habits of male-sterile transformants}

From among the seven most promising ASP constructs, we selected ASP108 and ASP304 (which generate could pollen-producing transformants), and ASP208 (which could generate pollen-less transformants) for further investigation; ASP208 was chosen because the pollen-less transformants derived from four other ASP constructs (ASP04, 204, 207, and 407) showed a very poor flowering rate compared with those derived from ASP208 in the preliminary investigation. A construct containing the $B O A 9$ promoter (Abe et al., 2018, Konagaya et al. 2008) in place of the ASP was used as a control. Wild type and transformants harboring the ASP108, ASP304, ASP208, or BoA9 constructs (about 10 individuals of each) were compared in terms of the following five survey items (1) number of days between heading and flowering, (2) number of days between the onset of flowering and the flowering peak, (3) flowering period, (4) flowering rate from 13:00 to 15:00 (the peak flowering time of wild type), and (5) flowering rate (Supplemental Fig. 2).

For each construct used, the flowering rate, the number of days between heading and flowering, and the flowering period of the individual transformants varied widely (Fig. 4, Supplementary Fig. 2), with the exception that the number 

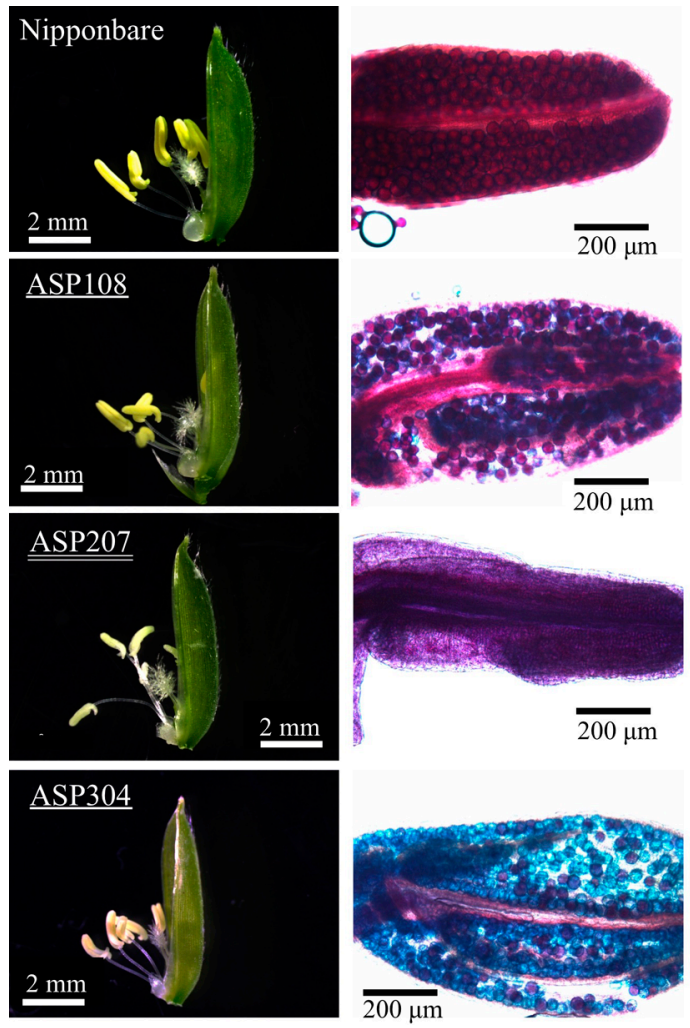

Fig. 3. Spikelets (left) and pollen grains (stained with Alexander's solution; right) of Nipponbare (wild type) and transformants. Left panels: Single underlines indicate the representative constructs that typically generate pollen-producing sterile transformants. Double underlines indicate the representative constructs that typically generate pollen-less sterile transformants. Right panels: blue and red staining indicates non-active and active pollen, respectively. of days between heading and flowering was consistently two days or less for ASP108 transformants (male-sterility with pollen grains), which was close to the one day or less observed for wild type (Fig. 5). For all constructs, most transformants showed a flowering rate from 13:00 to 15:00 of $0 \%-10 \%$, but some individual ASP304 transformants showed a flowering rate of $50 \%$ at this time (Supplemental Fig. 2C); a possible explanation for this is that, in this experiment, 5 out of 12 ASP304 transformants displayed incomplete male-sterility (Supplemental Fig. 2C, Supplemental Table 2). In the transformants harboring ASP208 or BoA9 (i.e., the pollen-less transformants), the flowering rates of many individuals were low compared to the rates observed for the pollen-producing transformants (Fig. 4). In BoA9, only two individuals (No. 5 and No. 7) could be examined in their peak of flowering, because the others showed no flowering or a remarkably low number of flowering spikelets per day, and the flowering time was too long (Supplemental Table 2).

\section{Discussion}

Comprehensive and effective screening of promoters in an expression profile database

In this study, we identified candidate anther-specific pro-
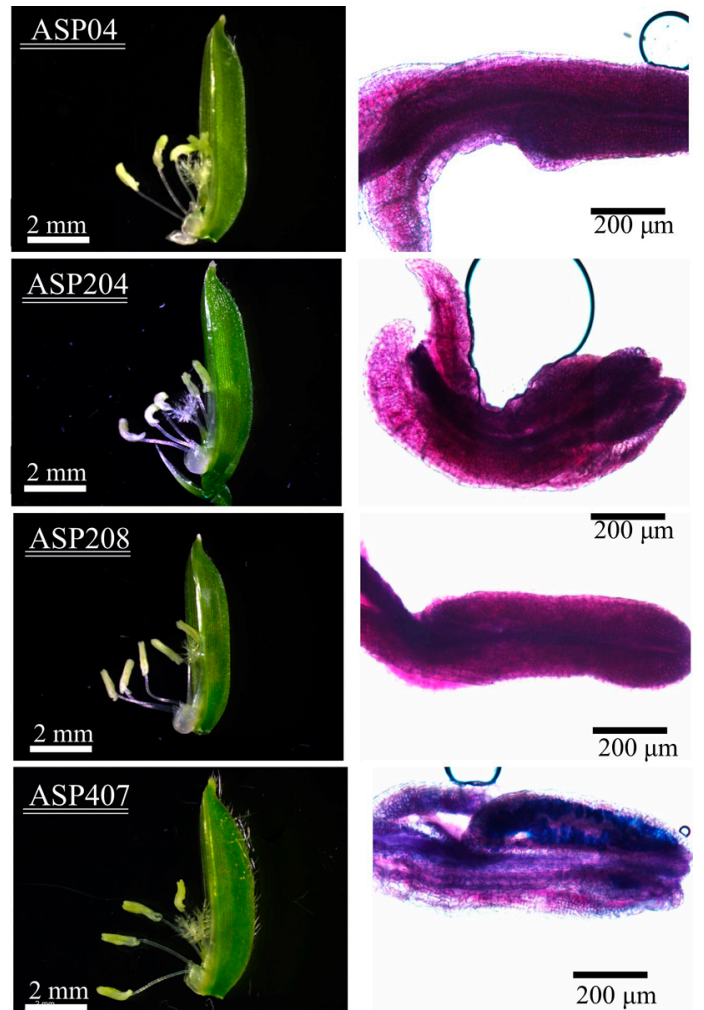

moters in silico by efficient screening of an expression profile database. This method has the following three advantages.

The first is that by targeting all the expressed genes in the database we can screen comprehensively for effective promoter candidates. The rice genome was sequenced in 2004 (IRGSP 2005), and since then various database tools such as 'RiceXPro', 'TOGO browser', 'RAP-DB', and 'Q-TARO' (Yonemaru et al. 2010) have been published. Here, we identified multiple ASP candidates of which ASP201 was identical to the promoter of PT42, which is registered in the US patent "Stamen-specific promoters from rice" (Michiels et al. 1997), and ASP407 was identical to the promoter of Osc6, which is listed as a "gene expressed in rice anthers" in Tsuchiya et al. (1992). Our identification of known antherspecific promoters in rice confirms the comprehensiveness of our strategy.

The second advantage is that by working in silico, it is possible to efficiently utilize research resources such as time, cost, and labor. Conventionally, to acquire tissuespecific promoters it is necessary to 1) extract RNA from the target tissue, 2) perform cDNA synthesis, 3) analyze the tissue-specificity of expression by Northern blotting etc. using the obtained cDNAs as probes, 4) screen clones of genomic fragments corresponding to cDNA from the genomic library, 5) evaluate the near-upstream sequences as specific 
Table 4. Cross-fertile of transformants

\begin{tabular}{|c|c|c|c|c|}
\hline Construct & $\begin{array}{c}\text { Individual } \\
\text { No. }\end{array}$ & $\begin{array}{l}\text { Total spikelets } \\
\text { (a) }\end{array}$ & $\begin{array}{l}\text { No. of set } \\
\text { seeds (b) }\end{array}$ & $\begin{array}{l}\text { Cross-fertility } \\
\text { (b/a) (\%) }\end{array}$ \\
\hline \multirow[t]{2}{*}{ ASP04 } & 1 & 41 & 4 & 9.8 \\
\hline & 2 & 107 & 1 & 0.9 \\
\hline \multirow[t]{3}{*}{ ASP108 } & 1 & 70 & 12 & 17.1 \\
\hline & 2 & 62 & 0 & 0.0 \\
\hline & 3 & 110 & 54 & 49.1 \\
\hline \multirow[t]{4}{*}{ ASP204 } & 1 & 31 & 15 & 48.4 \\
\hline & 2 & 26 & 6 & 23.1 \\
\hline & 3 & 25 & 1 & 4.0 \\
\hline & 4 & 17 & 7 & 41.2 \\
\hline \multirow[t]{4}{*}{ ASP207 } & 1 & 41 & 16 & 39.0 \\
\hline & 2 & 124 & 74 & 59.7 \\
\hline & 3 & 59 & 3 & 5.1 \\
\hline & 4 & 84 & 54 & 64.3 \\
\hline \multirow[t]{6}{*}{ ASP208 } & 1 & 68 & 20 & 29.4 \\
\hline & 2 & 105 & 17 & 16.2 \\
\hline & 3 & 78 & 25 & 32.1 \\
\hline & 4 & 184 & 22 & 12.0 \\
\hline & 5 & 75 & 13 & 17.3 \\
\hline & 6 & 109 & 15 & 13.8 \\
\hline \multirow[t]{2}{*}{ ASP304 } & 1 & 49 & 20 & 40.8 \\
\hline & 2 & 14 & 3 & 21.4 \\
\hline \multirow[t]{5}{*}{ ASP407 } & 1 & 155 & 82 & 52.9 \\
\hline & 2 & 98 & 57 & 58.2 \\
\hline & 3 & 33 & 12 & 36.4 \\
\hline & 4 & 35 & 9 & 25.7 \\
\hline & 5 & 79 & 34 & 43.0 \\
\hline \multirow[t]{4}{*}{ BoA9 } & 1 & 74 & 27 & 36.5 \\
\hline & 2 & 70 & 17 & 24.3 \\
\hline & 3 & 69 & 5 & 7.2 \\
\hline & 4 & 71 & 37 & 52.1 \\
\hline
\end{tabular}
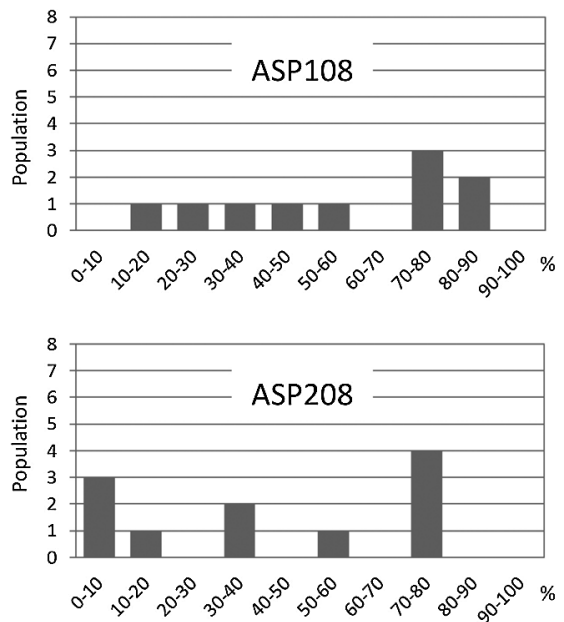

promoters, and so on. Here, by using genomic information resources including expression profiles, we could obtain specific candidate sequences by performing only PCR and subcloning, and we could proceed directly to the evaluation of each candidate to obtain suitable tissue-specific promoters.

The third advantage is that the strategy can be flexibly applied to the screening of promoters that are expressed in various tissues, environments, developmental stages, and/or daily time periods; for instance, in 'RiceXPro', datasets of expression at various time periods in a day at various developmental stages are available. In addition, stress response expression data in a database such as 'TENOR' (Kawahara et al. 2016) could be used to acquire stress-responsive promoters.

The utilization of a promoter that was identified by field transcriptomic analyses, and whose tissue-specificity was confirmed by using 'RiceXPro' (Okada et al. 2017), has been reported previously. However, the current study is the first to utilize this expression database for in silico screening to obtain tissue-specific promoters for use in transgenes. The results demonstrate that we could efficiently obtain desirable expression promoters by this strategy. Because of the above-mentioned three advantages of this strategy, this research will become an important milestone in attempts to acquire new tissue- or stage-specific promoters in the genomic era.

\section{Characterization of transgenic male-sterile rice}

In 'RiceXPro', the developmental stages of anther in 'Nipponbare' are described by their length, based on the
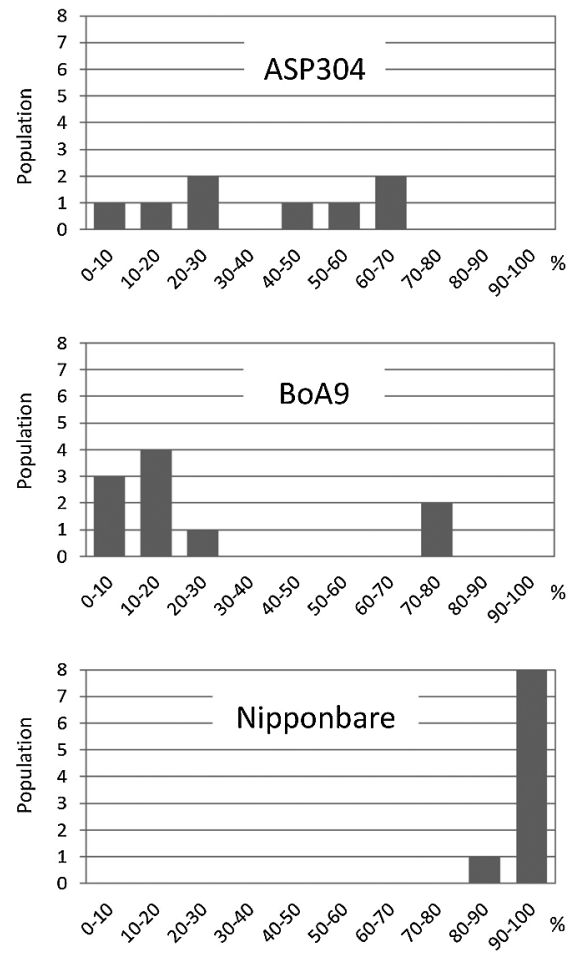

Fig. 4. Flowering rates of Nipponbare (wild type) and male-sterile transformants. The survey items are illustrated in Supplemental Fig. 2. 

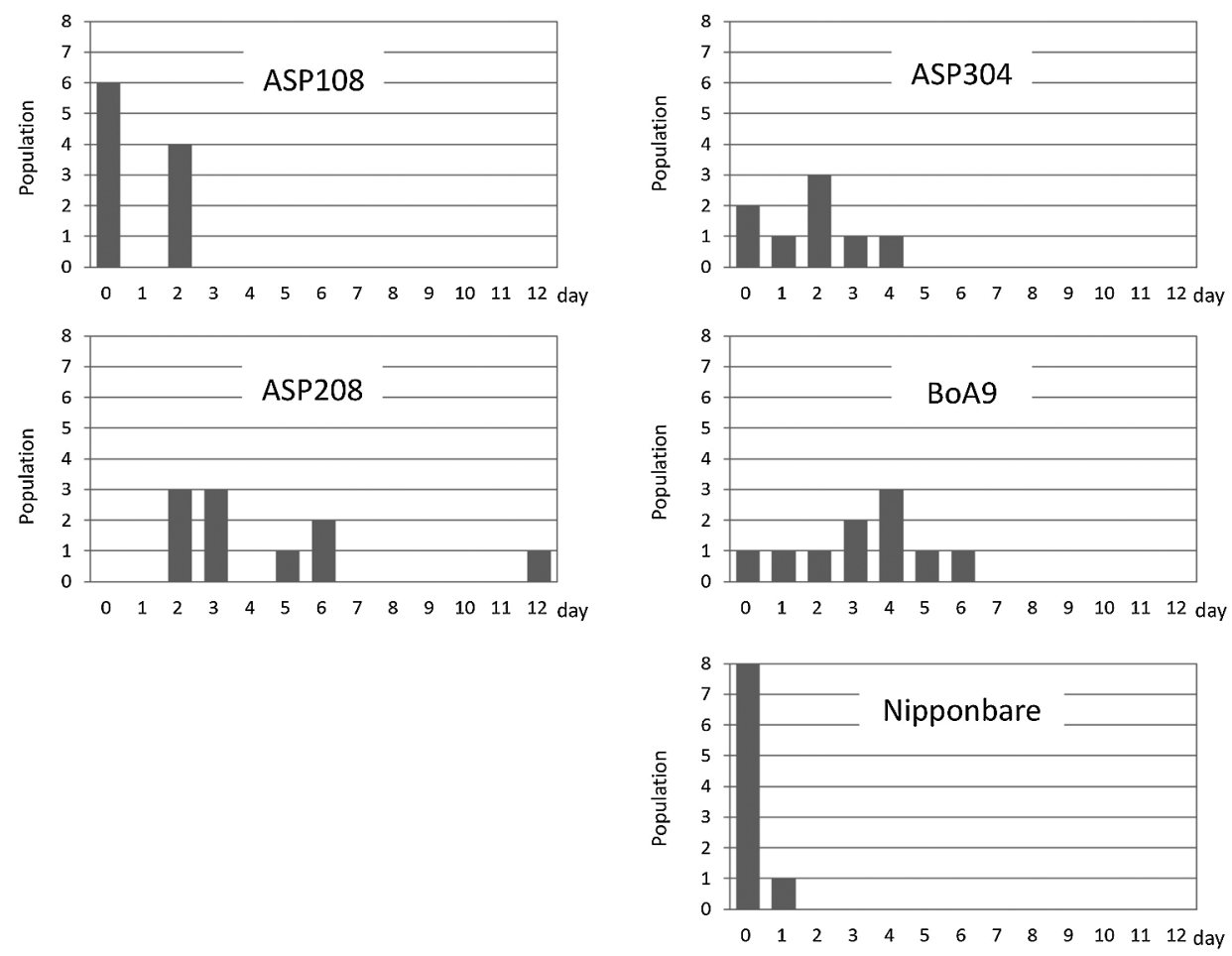

Fig. 5. Number of days between heading and flowering.

observations of Itoh et al. (2005): i.e., phase 1, formation of tapetum; phase 2, meiosis; phase 3 , formation of uninucleate gametophytes; and phase 4 , formation of mature pollen. Here, male-sterile transformants harboring ASPs predicted to be expressed mainly in the period from formation of tapetum to meiosis according to the RiceXPro data (e.g., ASP04, ASP204, and ASP207, Table 1) showed phenotypes of no pollen grains and anthers that were white and degenerated. The male-sterile transformants harboring ASP208, which is predicted to be expressed mainly in the period from meiosis to formation of uninucleate gametophytes, also showed the phenotype of no pollen grains. In contrast, male-sterile transformants harboring ASPs predicted to be highly expressed from formation of uninucleate gametophyte to mature pollen (e.g., ASP304 and ASP108, Table 1) showed phenotypes with pollen grains and anther shape and flowering characteristics similar to wild type (Table 3, Fig. 3). Our observation that the male-sterile transformants harboring ASP108, ASP109, ASP301, or ASP304 produced pollen grains with normal starch accumulation (Fig. 3), raises the possibility that their pollen tube could not elongate normally, as in the CW-cytoplasmic male-sterility line (Fujii and Toriyama 2005).

Our finding that flowering characteristics differed largely between individuals within the transformant population of each ASP construct indicates that it is important to select individual transformants for creation of breeding lines. Among transformants harboring ASP304, 5 out of 12 individuals showed incomplete male-sterility indicating that attention may be required when using this promoter. In con- trast, transformants harboring ASP108 or ASP304 produced pollen grains, but showed more stable and higher rates of male-sterility than those harboring the known male-sterility promoter PT42 (ASP201, Supplemental Table 2). Our observation that many of the transformants harboring ASP108 showed better flowering characteristics than those containing the BoA9 promoter (Figs. 4, 5) indicates that ASP108 is a particularly promising promoter for the production of male-sterile plants that can efficiently produce outcrossed seeds. In addition, transformants with white and degenerate anthers without normal pollen (e.g., those harboring ASP208), have the advantage that it is easy to discriminate whether they are male-sterile or not at the time of flowering (Fig. 3), so their male-sterility can be reliably identified before pollination.

Many ASPs that are highly expressed during the formation of mature pollen according to 'RiceXPro' did not induce male-sterility in the current study. Since transgenes are commonly heterozygous in the $\mathrm{T}_{0}$ generation, when the barnase transgene is activated by the ASP during the formation of mature pollen, half of the pollen might be inactivated by the lethal gene, and half the pollen might remain active. Although these promoters are unsuitable for production of male-sterile plants, they might be effective for inactivating pollen and thus might be useful for the development of SPT (Seed Production Technology; https://www.pioneer.com/ home/site/about/news-media/media-kits/seed-productiontechnology/). 


\section{Future applications of ASP promoters in breeding}

In this study, a comprehensive screening of antherspecific expressed genes in the rice genome resulted in the discovery of seven promoters that can be used to induce male-sterility. Among these promoters, ASP108 appeared particularly promising for the development of male-sterile rice with excellent flowering habits. However, not all transformants carrying ASP108 exhibited excellent flowering habits; for instance, maximum flowering synchronization rate among the 10 individual transformants was about $15 \%$, therefore selection of individual transformants as a practical breeding tool is important. Furthermore, efforts to increase the outcrossed seed fertility ratio, for example, by introducing the stigma exsertion trait or open hull trait, would be advantageous in the future.

By using the ASPs obtained in this study, such as ASP108, efficient development of male-sterile rice has become possible. Based on this technology, we anticipate that it will be possible to develop male-sterile rice lines that are ideal for recurrent selection by introducing gene cassettes with a male-sterility sequence linked to a selectable marker gene. The current research should pave the way to a new era of crop breeding that can effectively utilize the genome information available for autogamous crops species, using transgenic male-sterility (Tanaka 2010).

\section{Acknowledgements}

We would like to thank Dr. M. Kuroda of Central Region Agricultural Research Center, NARO, Japan and Dr. H. Yoshida of Institute of Agrobiological Sciences, NARO for their productive advice on vector construction, and Dr. Y. Sato and Dr. Y. Nagamura Institute of Crop Science, NARO for their technical advices of utilization of RiceXPro. We also thank J. Shioda, Y. Iguchi, M. Yamao, and Y. Niizeki from Institute of Crop Science, NARO, for their technical assistance. We also thank Dr. Nobuhiro Suzuki of Sophia University, Japan, for revision of the English in the manuscript. This work was partially supported by a grant from the Ministry of Agriculture, Forestry and Fisheries of Japan (Genomics for Agricultural Innovation GMO-1001).

\section{Literature Cited}

Abe,K., M.Oshima, M.Akasaka, K.Konagaya, Y.Nanasato, A. Okuzaki, Y.Taniguchi, J. Tanaka and Y. Tabei (2018) Development and characterization of transgenic dominant male sterile rice toward an outcross-based breeding system. Breed. Sci. 68: 248-257.

Alexander, M.P. (1969) Differential staining of aborted and nonaborted pollen. Stain Technol. 44: 117-122.

Cao, B., Z. Huang, G. Chen and J. Lei (2010) Restoring pollen fertility in transgenic male-sterile eggplant by Cre/loxp-mediated sitespecific recombination system. Genet. Mol. Biol. 33: 298-307.

De Block, M., D. Debrouwer and T. Moens (1997) The development of nuclear male sterility system in wheat. Expression of the barnase gene under the control of tapetum specific promoters. Theor. Appl. Genet. 95: 125-131.
Don, R.H., B.J.Cox, B.J. Wainwright and J.S. Mattick (1991) 'Touchdown' PCR to circumvent spurious priming during gene amplification. Nucleic Acids Res. 19: 4008

Fujii, S. and K. Toriyama (2005) Molecular mapping of the fertility restorer gene for ms-CW-type cytoplasmic male sterility of rice. Theor. Appl. Genet. 111: 696-701.

Fujimaki,H. (1980) Recurrent population improvement in rice breeding facilitated with male sterility. Gamma Field Symposia 19: 91102.

García-Ruiz,A., J.B.Cole, P.M.Van Raden, G.R.Wiggans, F.J.RuizLópez and C.P.Van Tassell (2016) Changes in genetic selection differentials and generation intervals in US Holstein dairy cattle as a result of genomic selection. Proc. Natl. Acad. Sci. USA 113 E3995-4004.

García-Sogo,B., B.Pineda, E. Roque, T.Antón, A.Atarés, M. Borja, J.P. Beltrán, V. Moreno and L.A.Cañas (2012) Production of engineered long-life and male sterile Pelargonium plants. BMC Plant Biol. 12: 156-171.

International Rice Genome Sequencing Project (2005) The map-based sequence of the rice genome. Nature 436: 793-800.

Itoh, J., K. Nonomura，K. Ikeda，S. Yamaki，Y. Inukai， H. Yamagishi, H. Kitano and Y. Nagato (2005) Rice plant development: from zygote to spikelet. Plant Cell Physiol. 46: 23-47.

Jagannath, A., P. Bandyopadhyay, N.Arumugam, V. Gupta, P.K. Burma and D.Pental (2001) The use of a spacer DNA fragment insulates the tissue-specific expression of a cytotoxic gene (barnase) and allows high-frequency generation of transgenic male sterile lines in Brassica juncea L. Mol. Breed. 8: 11-23.

Kawahara, Y., Y. Oono, H. Wakimoto, J. Ogata, H. Kanamori, H. Sasaki, S. Mori, T.Matsumoto and T.Itoh (2016) TENOR: Database for comprehensive mRNA-seq experiments in rice. Plant Cell Physiol. 57: e7.

Konagaya, K., S.Ando, S.Kamachi, M.Tsuda and Y.Tabei (2008) Efficient production of genetically engineered, male-sterile Arabidopsis thaliana using anther-specific promoters and genes derived from Brassica oleracea and B. rapa. Plant Cell Rep. 27: 1741-1754.

Kuroda, M., M. Kimizu and C. Mikami (2010) A simple set of plasmids for the production of transgenic plants. Biosci. Biotechnol. Biochem. 74: 2348-2351.

Lu, G.H., H.T.Sun, J.L.Zhang and M.M.Hong (2000) Induction of male sterility by the integration of chimeric RTS-barnase gene into rice (Oryza sativa L.) genome. Acta Physiol. Sinica 26: 171-176.

Luo, Z. and Z. Chen (2007) Improperly terminated, unpolyadenylated mRNA of sense transgenes is targeted by RDR6-mediated RNA silencing in Arabidopsis. Plant Cell 19: 943-958.

Mariani,C., M.DeBeuckeleer, J.Truettner, J.Leemans and R.B. Goldberg (1990) Induction of male sterility in plants by a chimaeric ribonuclease gene. Nature 347: 737-741.

Meuwissen, T.H.E., B.J. Hayes and M.E. Goddard (2001) Prediction of total genetic value using genome-wide dense marker maps. Genetics 157: 1819-1829.

Michiels, F., S. Morioka, T. Scheirlinck and T.Komari (1997) Stamenspecific promoters from rice. U.S. Patent No. 5,639,948.

Nagamura, Y., B.A.Antonio, Y. Sato, A. Miyao, N. Namiki, J. Yonemaru, H. Minami, K. Kamatsuki, K. Shimura, Y.Shimizu et al. (2011) Rice TOGO Browser: a platform to retrieve integrated information on rice functional and applied genomics. Plant Cell Physiol. 52: 230-237.

Ni,F., J.Qi, Q.Hao, B.Lyu, M.Luo, Y.Wang, F.Chen, S.Wang, C. Zhang, L.Epstein et al. (2017) Wheat Ms2 encodes for an orphan 
protein that confers male sterility in grass species. Nat. Commun. 8: 15121

Okada, R., Y.Nemoto, N. Endo-Higashi and T. Izawa (2017) Synthetic control of flowering in rice independent of the cultivation environment. Nat. Plants 3: 17039.

Ozawa, K. and F. Takaiwa (2010) Highly efficient Agrobacteriummediated transformation of suspension-cultured cell clusters of rice (Oryza sativa L.). Plant Sci. 179: 333-337.

Paddon, C.J. and R.W.Hartley (1985) Cloning, sequencing and transcription of an inactivated copy of Bacillus amyloliquefaciens extracellular ribonuclease (barnase). Gene 40: 231-239.

Sakai,H., S.S.Lee, T.Tanaka, H.Numa, J.Kim, T.Kawahara, H. Wakimoto, C. Yang, M. Iwamoto, T. Abe et al. (2013) Rice annotation project database (RAP-DB): an integrative and interactive database for rice genomics. Plant Cell Physiol. 54: e6.

Sato, Y., B.A.Antonio, N. Namiki, H.Takehisa, H.Minami, K. Kamatsuki, K. Sugimoto, Y.Shimizu, H. Hirochika and Y. Nagamura (2011) RiceXPro: a platform for monitoring gene expression in japonica rice grown under natural field conditions. Nucleic Acids Res. 39: D1141-D1148.

Sato, Y., H. Takehisa, K. Kamatsuki, H. Minami, N. Namiki, H. Ikawa, H. Ohyanagi, K. Sugimoto, B.A.Antonio and Y.Nagamura (2013) RiceXPro Version 3.0: expanding the informatics resource for rice transcriptome. Nucleic Acids Res. 41: D1206-D1213.

Song, Z.P., B.R. Lu and J.K. Chen (2001) A study of pollen viability and longevity in Oryza rufipogon, O. sativa, and their hybrids. Int. Rice Res. Notes 26: 31-32.

Sun, Q., Y.Zhang, T. Rong, S. Dong, D. Ma and C. Zhang (2008) Establishment of transgenic acceptor and transformation of barnase gene by particle gun in maize inbred line 18-599 (white). Front. Agric. China 2: 37-43.
Tamaru, N. (1994) Breeding studies on genetic male sterility and hybrid sterility in rice (Oryza sativa L.). Mem. Fac. Agr. Hokkaido Univ. 19: 203-256.

Tanaka, J. (2010) Transgenic male sterility permits efficient recurrent selection in autogamous crops. Crop Sci. 50: 1124-1127.

Tanaka, J. and S.Ikeda (2002) Rapid and efficient DNA extraction method from various plant species using diatomaceous earth and a spin filter. Breed. Sci. 52: 151-155.

Tanaka, J. and Y. Tabei (2014) Effort to increase breeding efficiency by reproduction control using NBT-SPT (seed production technology) process, reverse breeding, early flowering in fruit trees, and TMS recurrent selection in autogamous crops. Seibutsu-no-Kagaku Iden 68: $117-124$.

Tanaka, J., T. Hayashi and H.Iwata (2016) A practical, rapid generation-advancement system for rice breeding using simplified biotron breeding system. Breed. Sci. 66: 542-551.

Tsuchiya, T., K. Toriyama, M.E. Nasrallah and S. Ejiri (1992) Isolation of genes abundantly expressed in rice anthers at the microspore stage. Plant Mol. Biol. 20: 1189-1193.

Yang, K., Y.Chen, M. Shi, R. Converse, X. Chen, B.Zhao, Y.Zhang and J. Lv (2017) A novel dominant rice male sterility mutant, OsDMS-1, simultaneously controlled by independent loci on chromosomes 1 , 2, and 3. Mol Breed. 37: 25.

Yonemaru, J., T. Yamamoto, S. Fukuoka, Y.Uga, K. Hori and M. Yano (2010) Q-TARO: QTL Annotation Rice Online database. Rice 3: 194-203.

Zhang, C., K.H.Norris-Caneda, W.H.Rottmann, J.E. Gulledge, S. Chan, B.Y.Kwan, A.M.Thomas, L.C.Mandel, R.T.Kothera, A.D. Victor et al. (2012) Control of pollen-mediated gene flow in transgenic trees. Plant Physiol. 159: 1319-1334. 